\title{
Delamination Modeling and Assessment for Composite Laminated Plates using Particle Swarm Optimization (PSO)
}

\author{
Nabil H. Hadi, PhD \\ Mechanical Engineering Department, \\ College of Engineering. \\ University of Baghdad. \\ Baghdad, Iraq.
}

\begin{abstract}
This work reports on the finite element method and particle swarm optimization method (PSO) for modeling and identification of delamination in composite material. A first order shear deformation using nine nodded isoparametric quadratic element with a simple multiple delamination model is used to develop the finite element analysis procedure. Delamination is the most common failure mechanism in composite structures. These damage causes changes in the physical properties by reducing the stiffness of the structure which leads to changes in modal parameters such as the frequencies, mode shapes, and modal damping factors. Identify the presence of damage and assess its size and location from a few lower frequency modes in modal test is the main objective of this study. Particle Swarm Optimization delamination detection based on the utilization of the changes in modal parameters as the objective function successfully identify the presence, location, and relative area size in composite laminated plates.
\end{abstract}

\section{Keywords}

composite laminates, Vibration Monitoring, Delamination, Damage Detection, Structural Health Monitoring, Finite element analysis

\section{INTRODUCTION}

Composite materials structures have been increasingly used due to their high strength and stiffness to weight ratios, ease of manufacturing, light weight, ability to withstand fatigue and corrosion, and cost effectiveness that associated them with high performance and significant weight-saving compared to traditional materials.

Despite their apparent superiority to metals, such composite materials in service can suffer from a number of failure mechanisms, such as ply or fiber breakage, matrix cracking[1] and delaminations of which delamination is most common, easily exposed to damage and it may propagate, thus reducing the life of the structure.

Essentially, delamination is the greatest weakness of composite laminates. Delamination or interlaminar damage is the separation of the laminate plies as a result of low strength.

This could lead to loss of structural integrity [2]. Delaminations can easily spread throughout the whole laminate of a composite structure upon repeated loading causing costly and/or disastrous failures when undetected.
Online vibration based monitoring using shifts in natural frequencies can provide early warning of occurrence of delamination[1].

Generally dynamic analysis of the structure can be done by Finite Element Method (FEM). In structural engineering, dynamic analysis of structures can be divided into two categories, one is related with the low frequency loading categorized as structural dynamic problems and another related with high frequency loading categorized as wave propagation problems[3].

Most of the structures of dynamic analysis come under structural dynamics. In structural dynamics problems, the solution can be determined either by system parameters such as natural frequencies and mode shapes or in terms of simulated response of the system to the external excitation such as initial displacements support motion and applied load etc.[3].

Since frequency shifts can be measured more accurately and reliably without requiring a large number of sensors to be mounted on the structure. However, one of the disadvantages of frequency monitoring is that, while it readily indicates the presence of damage, identification of the location and severity of damage requires complex information processing using mathematical or intelligent inverse algorithms tools such as Neural Networks and Genetic Algorithm [4] [5] [6]. Research on application of such techniques to detection of delaminations in composites is still ongoing and has so far yielded mixed results.[6]. Rytter, and Kirkegaard [4] presented a new model-based delamination detection methodology for laminated composite plates and its performance was studied both numerically and experimentally. In addition to numerical demonstrations, the proposed novel damage detection method is experimentally demonstrated using the scanning laser vibrometer and a two dimensional E-glass/epoxy composite panel.

In [7] The applications of the proposed methodology to an Eglass/epoxy symmetric composite panel composed of 16 plies withed lamination damage were demonstrated both numerically and experimentally. The modal parameters are used in the subset selection analysis, from which the delamination damage is successfully detected and located. 
Vinh[8] presented an isogeometric computational framework for modeling delamination of two and three dimensional composite laminates. Used isogeometric cohesive elements for modeling two and three dimensional delaminated composite structures. they exploit the knot insertion algorithm offered by NURBS (Non Uniform Rational B-splines) to generate cohesive elements along delamination planes in an automatic fashion.

\section{There are many advantages to using a frequency response}

\section{method in a Structural Health Monitoring}

(SHM) system; they can be implemented cheaply, they can be light and conformal, and they can provide good insight as to the global condition of the system.

The limitations are that they provide little information about the local damage area unless large quantities of sensors are used along with accurate numerical models; and then it can be argued that damage large enough to be detected globally may already be critical in many structures. [9].

Kyoung and et al.[10] Analytically and experimentally studied. A Lamb wave-based quantitative identification technique for delamination in composite structures. Propagation of Lamb waves in composite plates was evaluated using dynamic FEM analysis. The study was carried out to assess damages in composite plate by fusing information from multiple sensing paths of the embedded network. It was based on the Hilbert transform, signal correlation and probabilistic searching. Seth [9] For the purpose of the optimization problem of damage detection, a FE model updating based on multi-objective intelligent algorithm was carried out . A new multi-objective function defined by natural frequencies and accumulative modal assurance criterion (MAC) was formulated. The results show that the combination of the new multi-objective function and multi-objective differential evolution optimization (DEMO) algorithm has the highest calculation accuracy and efficiency. Doing an attempts to summarize the recent trends and progress of vibration signatures obtained from damaged structures under dynamic conditions. This study focuses on the identification of the presence, location, and size of delamination in the laminated composite plates by extracting the modal parameters obtained from the SLV measurement system(Laser Vibrometer). Oruganti [11] Use the vibration response of a composite plate to detect and localize delamination defect based on the frequency response and modal analysis. The features extracted are used as the input data in an artificial intelligence scheme to identify the severity of the damages. Experiments were then conducted to validate the developed model. Nasiri[12] their study was to applied the Genetic algorithm with fem delamination modeling for the quantitative identification of delamination in composite plates. Damage assessment methods based on frequency changes require frequency measurements to be taken both on the undamaged and damaged composite plate structures. This is true for methods using mode shapes and their derivatives. Numerical test cases demonstrate that delaminations of small size can be identified and assessed using the present method. In this paper the reliability of the frequency response of a composite structure with the help of PSO method in a Structural Health Monitoring system has been investigated.

\section{FINITE ELEMENT MODELING AND ANALYSIS OF LAMINATED COMPOSITE PLATES}

The higher-order shear deformation theories have also been employed for buckling analysis of laminated composite platesThe mechanical behavior of a moderately thick laminate plate is described through a First order Shear Deformation Theory (FSDT). The theory takes into account in-plane deformation, bending and first-order shear effects. Laminated plate refers is a body, constituted by $\mathrm{n}$ layers of different mechanical characteristics, occupying the region: [13]

$O=\left\{(x, y, z) \in \frac{R^{3}}{z} \in\left(-\frac{h}{2}, \frac{h}{2}\right),(x, y) \in A \in R^{2}\right\}$

where the plane $\mathrm{z}=0$ identifies the mid-surface $\mathrm{A}$ of the un deformed plate. The thickness $h$ is assumed to be small compared to the in-plane dimensions. Basic elements of the FSDT are the transverse stress in the thickness of the plate $\sigma_{z}$ is null

and the straight lines orthogonal to the mid plane are inextensible and remain straight after deformation. It is interesting to recall that in a general three-dimensional elastic theory the simultaneous presence of these

two statements is formally correct and, in fact, they can be rationally introduced to obtain the FSDT for isotropic homogeneous plate[13]. All materials are linear, homogeneous, orthotropic and made of elastic laminated composite materials;

This study only focus on the linear elastic behaviors of fiber reinforced composites of transversely isotropic type. The frequency response is determine through the finite element method formulation of fiber reinforced composites. Besides, each element has nine nodes comprising five degree of freedoms at each node.

A rectangular Cartesian coordinate system $\mathrm{x}, \mathrm{y}$ and $\mathrm{z}$ is used to describe the infinitesimal deformations of a n-layer laminated composite material, shown in Fig.1a, Consider a $K$ layered plate of in-plane dimensions $a, b$ and thickness $h$. The present First order Shear Deformation Theory (FSDT) is developed with the assumption of the displacement model of the following form: The displacements $u, v$ and $w$ at any point $(x, y, z)$ in the laminate are given by[14]

$u(x, y, z)=u_{o}+z \emptyset_{x}(x, y, z)$

$v(x, y, z)=v_{o}+z \emptyset_{y}(x, y, z)$

$w(x, y, z)=w_{o}(x, y, z)$

in which uo, vo and wo are the in-plane and transverse displacements of a point $(\mathrm{x}, \mathrm{y})$ on the mid-plane respectively and $\emptyset x, \varnothing y$ are the rotations of normal to mid-plane about $y$ and $x$ axes respectively, owing to bending only.

The strains associated with the displacement field (1) the strain are given by:

$\varepsilon_{x x}=\frac{\partial u}{\partial x}, \varepsilon_{y y}=\frac{\partial v}{\partial y}, \varepsilon_{z z}=\frac{\partial w}{\partial z}, \gamma_{x y}=\left(\frac{\partial u}{\partial y}+\frac{\partial v}{\partial x}\right)$,
$\gamma_{z x}=\left(\frac{\partial w}{\partial x}+\emptyset_{x}\right), \gamma_{y z}=\left(\frac{\partial w}{\partial y}+\emptyset_{y}\right)$

Using the expressions for $\mathrm{u}, \mathrm{v}, \mathrm{w}$ from eq. (1) with equation (2) gives: 
$\varepsilon_{x x}=\frac{\partial u_{o}}{\partial x}+z \frac{\partial \emptyset_{x}}{\partial x}, \varepsilon_{y y}=\frac{\partial v_{o}}{\partial y}+z \frac{\partial \emptyset_{y}}{\partial y}, \varepsilon_{z z}=0$

$\gamma_{x y}=\left(\frac{\partial u_{o}}{\partial y}+z \frac{\partial \emptyset_{x}}{\partial y}\right)+\left(\frac{\partial v_{o}}{\partial x}+z \frac{\partial \emptyset_{y}}{\partial x}\right), \gamma_{z x}=\left(\frac{\partial w_{o}}{\partial x}+\emptyset_{x}\right)$,

$\gamma_{y z}=\left(\frac{\partial w_{o}}{\partial y}+\emptyset_{y}\right)$

(3)

The above equations can be written in matrix form as[14]

$$
\left\{\begin{array}{l}
\varepsilon_{x x} \\
\varepsilon_{y y} \\
\gamma_{x y} \\
\gamma_{z x} \\
\gamma_{y z}
\end{array}\right\}=\left\{\begin{array}{c}
\frac{\partial u_{o}}{\partial x} \\
\frac{\partial v_{o}}{\partial y} \\
\frac{\partial u_{o}}{\partial y}+\frac{\partial v_{o}}{\partial x} \\
\frac{\partial w_{o}}{\partial x}+\emptyset_{x} \\
\frac{\partial w_{o}}{\partial y}+\emptyset_{y}
\end{array}\right\}+z\left\{\begin{array}{c}
\frac{\partial \emptyset_{x}}{\partial x} \\
\frac{\partial \emptyset_{y}}{\partial y} \\
\frac{\partial \emptyset_{x}}{\partial y}+\frac{\partial \emptyset_{y}}{\partial x} \\
0 \\
0
\end{array}\right\} .
$$

Or in the form:

$$
\left\{\begin{array}{l}
\varepsilon_{x x} \\
\varepsilon_{y y} \\
\gamma_{x y} \\
\gamma_{x z} \\
\gamma_{y z}
\end{array}\right\}=\left\{\begin{array}{l}
\varepsilon_{x x}^{o} \\
\varepsilon_{y y}^{o} \\
\gamma_{x y}^{o} \\
\gamma_{x z}^{o} \\
\gamma_{y z}^{o}
\end{array}\right\}+z\left\{\begin{array}{c}
k_{x} \\
k_{y} \\
k_{x y} \\
0 \\
0
\end{array}\right\}
$$

\subsection{Constitutive Relations}

The Stress-Strain relations for a typical lamina $\mathrm{k}$ with reference to the lamina co-ordinate axes (1-2-3) are given by

$\left\{\begin{array}{l}\sigma_{1} \\ \sigma_{2} \\ \sigma_{12}\end{array}\right\}^{k}=\left[\begin{array}{ccc}Q_{11} & Q_{12} & 0 \\ Q_{21} & Q_{22} & 0 \\ 0 & 0 & Q_{33}\end{array}\right]^{k}\left\{\begin{array}{c}\varepsilon_{1} \\ \varepsilon_{2} \\ \varepsilon_{12}\end{array}\right\}^{k}$
$\left\{\begin{array}{l}\tau_{23} \\ \tau_{13}\end{array}\right\}^{k}=\kappa\left[\begin{array}{cc}Q_{44} & 0 \\ 0 & Q_{55}\end{array}\right]^{k}\left\{\begin{array}{l}\gamma_{23} \\ \gamma_{13}\end{array}\right\}^{k}$

(5)

The Qij's are the plane stress elastic constants of the kth lamina and the following relations hold between these and the engineering constants.

$$
\begin{gathered}
Q_{11}=\frac{E_{1}}{1-v_{12} v_{11}}, \quad Q_{22}=\frac{E_{2}}{1-v_{12} v_{21}}, \quad Q_{12}=\frac{v_{12} E_{2}}{1-v_{12} v_{21}}=\frac{v_{21} E_{1}}{1-v_{12} v_{21}}, \\
Q_{33}=G_{12}, \quad Q_{44}=G_{23}, Q_{25}=G_{13} .
\end{gathered}
$$

And $\kappa$ refers to the Shear Correction Factor used in FSDT. Normally its value is $5 / 6$.

\subsection{Stress - Strain relations for a Lamina of Arbitrary Orientation}

The transformation equations for expressing stresses in 1-2 principal coordinate system in terms of stresses in $x-y$ coordinate system. [14]

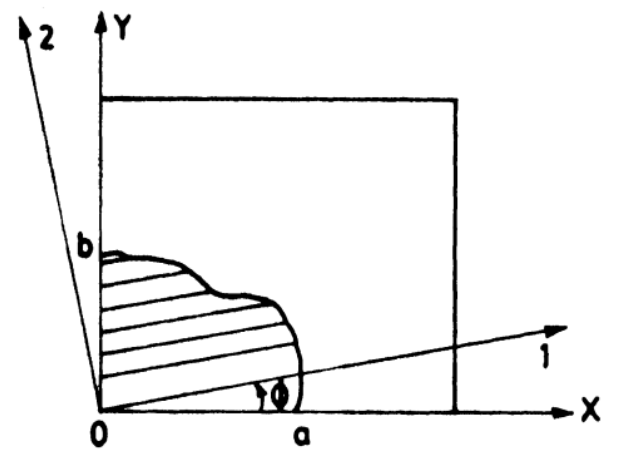

(a)

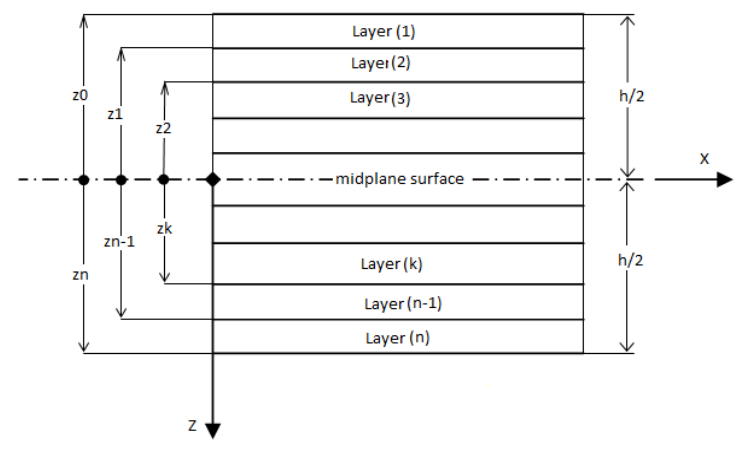

(b)

Fig 1: Plate axes and layer details. [15]

$\left\{\begin{array}{c}\sigma_{1} \\ \sigma_{2} \\ \sigma_{x y}\end{array}\right\}=\left[\begin{array}{ccc}\cos ^{2} \theta & \sin ^{2} \theta & 2 \cos \theta \sin \theta \\ \sin ^{2} \theta & \cos ^{2} \theta & -2 \cos \theta \sin \theta \\ -\sin \theta \cos \theta & \sin \theta \cos \theta & \cos ^{2} \theta-\sin ^{2} \theta\end{array}\right]\left\{\begin{array}{c}\sigma_{x} \\ \sigma_{y} \\ \sigma_{x y}\end{array}\right\}$ (7)

Then if the principal axes of the lamina are rotated to coincide with the global $\mathrm{x}$

and y axes, the transformation of stiffness matrix is expressed in equation (7).

$(\bar{Q})_{k}=$
$\left[\begin{array}{lcc}m^{2} & n^{2} & -2 m n \\ n^{2} & m^{2} & 2 m n \\ m n & -m n & m^{2}-n^{2}\end{array}\right]\left(Q_{i j}\right)_{k}\left[\begin{array}{ccc}m^{2} & n^{2} & -2 m n \\ n^{2} & m^{2} & 2 m n \\ m n & -m n & m^{2}-n^{2}\end{array}\right]$,
$i, j=1,2,6$

$(\bar{Q})_{k}=\left[\begin{array}{cc}m & -n \\ n & m\end{array}\right]\left(Q_{i j}\right)_{k}\left[\begin{array}{cc}m & -n \\ n & m\end{array}\right], \quad \quad i, j=4,5$.

Where $m=\cos \theta, n=\sin \theta$

Where $\Theta$ is the angle from the $\mathrm{x}$-axis to the axis 1 .

Hence, the stress strain relationship for angle ply is obtained as

$\left\{\begin{array}{c}\sigma_{x} \\ \sigma_{y} \\ \sigma_{x y}\end{array}\right\}^{k}=\left[\begin{array}{lll}\bar{Q}_{11} & \bar{Q}_{12} & \bar{Q}_{16} \\ \bar{Q}_{21} & \bar{Q}_{22} & \bar{Q}_{26} \\ \bar{Q}_{16} & \bar{Q}_{26} & \bar{Q}_{66}\end{array}\right]^{k}\left\{\begin{array}{c}\varepsilon_{x} \\ \varepsilon_{y} \\ \varepsilon_{x y}\end{array}\right\}$

(9)

$\left\{\begin{array}{l}\tau_{x z} \\ \tau_{y z}\end{array}\right\}^{k}=\left[\begin{array}{ll}\bar{Q}_{44} & \bar{Q}_{45} \\ \bar{Q}_{54} & \bar{Q}_{55}\end{array}\right]^{k}\left\{\begin{array}{l}\gamma_{x z} \\ \gamma_{y z}\end{array}\right\}^{k}$ 
By integrating the stresses in equations 9 and 10 over the thickness of the laminated plate the elasticity matrix of the undelaminated composite plate is given by

$[D]=\left[\begin{array}{ccc}A_{i j} & B_{i j} & 0 \\ B_{i j} & D_{i j} & 0 \\ 0 & 0 & A_{s i j}\end{array}\right]$,

where $[\mathrm{A}],[\mathrm{B}]$ and $[\mathrm{D}]$ are the extension, bending-extension coupling, and bending stiffness coefficients of the composite laminate, respectively.

$\left[\mathrm{A}_{\mathrm{ij}} \mathrm{B}_{\mathrm{ij}} \mathrm{D}_{\mathrm{ij}}\right]=\sum_{\mathrm{k}=1}^{\mathrm{n}} \int_{\mathrm{z}_{\mathrm{k}-1}}^{\mathrm{z}_{\mathrm{k}}}\left[\overline{\mathrm{Q}}_{\mathrm{ij}}\right]_{\mathrm{k}}\left(1, \mathrm{z}, \mathrm{z}^{2}\right) \mathrm{dz}, \quad \mathrm{i}, \mathrm{j}=1,2,6$.

And

$\left[\mathrm{A}_{\mathrm{sij}}\right]=\sum_{\mathrm{k}=1}^{\mathrm{n}} \int_{\mathrm{z}_{\mathrm{k}-1}}^{\mathrm{z}_{\mathrm{k}}} \kappa\left[\overline{\mathrm{Q}}_{\mathrm{ij}}\right]_{\mathrm{k}}\left(1, \mathrm{z}, \mathrm{z}^{2}\right) \mathrm{dz}, \quad \mathrm{i}, \mathrm{j}=4,5$.

A nine noded isoparametric quadratic plate bending element ,fig.(2) with five degrees of freedom at each node (three translation and two rotations) is employed wherein the shape functions are as follows [1] [16]

Corner Nodes

$N_{i}=\frac{1}{4} \xi\left(\xi+\xi_{i}\right) \eta\left(\eta+\eta_{i}\right) \quad i=1,2,3,4$

Mid-Side Nodes

$$
\begin{aligned}
N_{i}=\frac{1}{2}\left[1-\left(\xi \xi_{i}\right)^{2}-\left(\eta \eta_{i}\right)^{2}\right] \\
\\
{\left[\xi \xi_{i}\left(1+\left(\xi \xi_{i}\right)\right)+\eta \eta_{i}\left(1+\left(\eta \eta_{i}\right)\right)\right] } \\
\quad i=5,6,7,8
\end{aligned}
$$

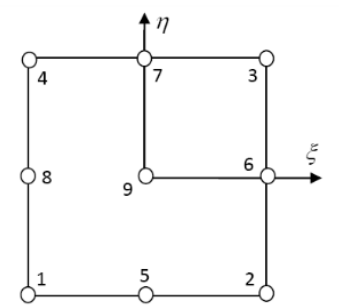

Fig 2: A nine node isoparametric element

\section{Middle Node}

$N_{i}=\left(1-\xi^{2}\right)\left(1+\eta^{2}\right) \quad i=9$

(14)

Where $\xi$ and $\eta$ are the local natural coordinates of the element $\xi_{i}$ and $\eta_{i}$ are the values at $i^{\text {th }}$ node.

The derivatives of the shape function $N_{i}$ with respect to x.y are expressed in term of their derivatives with respect to $\xi$ and $\eta$ by the following relationship

$\left[\begin{array}{c}N_{i, x} \\ N_{i, y}\end{array}\right]=[J]^{-1}\left[\begin{array}{c}N_{i, \xi} \\ N_{i, \xi}\end{array}\right]$

(15)

Where $\mathrm{J}$ is the Jacobean.
$[J]=\left[\begin{array}{ll}\frac{\partial y}{\partial \xi} & \frac{\partial y}{\partial \xi} \\ \frac{\partial y}{\partial \eta} & \frac{\partial y}{\partial \eta}\end{array}\right]$

The linear strain can be described in term of displacements as follow:

$\left\{\varepsilon_{e}\right\}=[B]\left\{\delta_{e}\right\}$

Where

$\left\{\delta_{e}\right\}=\left[\begin{array}{lll}u_{1} v_{1} w_{1} \emptyset_{x 1} \emptyset_{y 1} u_{2} v_{2} w_{2} \ldots \ldots & \ldots & u_{9} v_{9} w_{9} \emptyset_{x 9} \emptyset_{y 9}\end{array}\right]^{T}$, and $[B]$ is the strain displacement matrix

$[B]=\left[\left[B_{1}\right]\left[B_{2}\right] \ldots \ldots c \ldots c\left[B_{8}\right]\left[B_{9}\right]\right]$

$\left[B_{i}\right]=\left[\begin{array}{ccccc}\frac{\partial N_{i}}{\partial x} & 0 & 0 & 0 & 0 \\ 0 & \frac{\partial N_{i}}{\partial y} & 0 & 0 & 0 \\ \frac{\partial N_{i}}{\partial y} & \frac{\partial N_{i}}{\partial x} & 0 & 0 & 0 \\ 0 & 0 & 0 & \frac{\partial N_{i}}{\partial x} & 0 \\ 0 & 0 & 0 & 0 & \frac{\partial N_{i}}{\partial y} \\ 0 & 0 & 0 & \frac{\partial N_{i}}{\partial y} & \frac{\partial N_{i}}{\partial x} \\ 0 & 0 & \frac{\partial N_{i}}{\partial x} & N_{i} & 0 \\ 0 & 0 & \frac{\partial N_{i}}{\partial y} & 0 & N_{i}\end{array}\right]$

The element stiffness $\left[K_{e}\right]$ and consistent mass matrices $\left[M_{e}\right]$ are given by

$$
\begin{aligned}
& {\left[K_{e}\right]=\int_{-1}^{1} \int_{-1}^{1}[B]^{T}[D][B] J d \xi d \eta} \\
& {\left[M_{e}\right]=\int_{-1}^{1} \int_{-1}^{1}[N]^{T}[\rho][N] J d \xi d \eta}
\end{aligned}
$$

Where

$$
\begin{aligned}
& {[N]=\left[\begin{array}{ccccc}
N_{i} & 0 & 0 & 0 & 0 \\
0 & N_{i} & 0 & 0 & 0 \\
0 & 0 & N_{i} & 0 & 0 \\
0 & 0 & 0 & N_{i} & 0 \\
0 & 0 & 0 & 0 & N_{i}
\end{array}\right]} \\
& {[\rho]=\left[\begin{array}{ccccc}
P_{1} & 0 & 0 & P_{2} & 0 \\
0 & P_{1} & 0 & 0 & P_{2} \\
0 & 0 & P_{1} & 0 & 0 \\
P_{2} & 0 & 0 & P_{3} & 0 \\
0 & P_{2} & 0 & 0 & P_{3}
\end{array}\right]} \\
& \left(P_{1}, P_{2}, P_{3}\right)=\sum_{k=1}^{n} \int_{z_{k-1}}^{z_{k}}(\rho)_{k}\left(1, z, z^{2}\right) d z
\end{aligned}
$$

Assembling the element mass, stiffness and force matrices with respect to the common global axes, the resulting equilibrium equation is

$[M] \ddot{\delta}+[K] \delta=\{F\}$

For free vibration $[M] \ddot{\delta}+[K] \delta=0$ 
The eigenvalue equation for the free vibration analysis of laminated composite plate can expressed as

$\left([K]-\omega^{2}[M]\right)\{\delta\}=\{0\}$

Where $[M]$ and $[K]$ are the global mass and stiffness matrices , $\omega$ is the natural frequency and $\delta$ is the corresponding eigenvectors.

\subsection{Delamination Modeling}

In this work a simple two dimensional single delamination model proposed by Gim (1994)[17] and extended by Parhiet al. (2000) [18] will be used for the vibration of delaminated composite panels. In order to satisfy the compatibility and equilibrium requirements at the common delamination boundary, it is assumed that the in-plane displacement, transverse displacement and rotation at a common node for all the three sub laminates including the original one are identical applying multiple constraint condition at any arbitrary delamination boundary. It can be applicable to any general case of a laminated composite plate having multiple delaminations at any arbitrary location. Here, the delaminated area is assumed as the interface of two separate sub laminates bonded together along the delamination surface.

\section{A.Two Dimensional Delamination Model}

The two dimensional delamination model for the composite panels will be used in modeling delamination crack in the FEM. Figure(3) represents the cross-sectional view of a typical delamination crack tip where nodes of three plate elements meet together to form a common node.

The undelaminated region is modeled by plate element 1 of thickness $\mathrm{h}$, and the delaminated region is modeled by plate elements 2 and 3 whose interface contains the delamination (h2 and h3 are the thicknesses of the elements 2 and 3 respectively). The elements 1,2 and 3 are freely allowed to deform prior to imposition of the constraints conditions.

The nodal displacements of elements 2 and 3 at the crack tip are expressed as [10]

$$
\begin{aligned}
& u_{p}=u_{p}^{0}+\left(z-z_{p}^{0}\right) \emptyset_{x p} \\
& v_{p}=v_{p}^{0}+\left(z-z_{p}^{0}\right) \emptyset_{y p}
\end{aligned}
$$

$$
w_{p}=w
$$

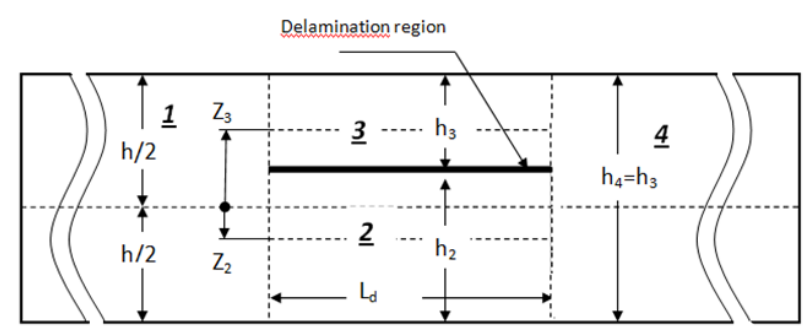

Fig 3: Cross-sectional of delamination crack tip where $u_{p}^{0}, v_{p}^{0}$ are the mid-plane displacement of the $p^{\text {th }}$ sub laminate and $z_{p}^{0}$ the distance between the mid-plane of the original laminate and the mid-plane of the $p^{t h}$ sub laminate. The above equation also holds good for element 1 and $z_{1}^{0}$ equal to zero. To satisfy the compatibility condition at the delamination's boundary, the transverse displacements and rotations at a common node for all the three sub laminates including the original one are identical and this leads to asymmetric stiffness matrices. The transverse displacements and rotations at a common node in the delamination region must have values expressed as below

$w_{1}=w_{2}=w_{3}=w$

$\emptyset_{x 1}=\emptyset_{x 2}=\emptyset_{x 3}=\emptyset_{x}$

$\emptyset_{y 1}=\emptyset_{y 2}=\emptyset_{y 3}=\emptyset_{y}$

Applying constraint conditions that at any delamination boundary, the transverse displacements and rotations must have the same values at a common node, the midpoint displacements of any sub-laminate $p^{t h}$ can be generalized as

$u_{p}^{0}=u^{0}+z_{p}^{0} \emptyset_{x}$

$v_{p}^{0}=v^{0}+z_{p}^{0} \emptyset_{y}$

From eq(24) the midplane strain components of the $p^{\text {th }}$ sublamiate are

$\left\{\begin{array}{l}\varepsilon_{x x}^{o} \\ \varepsilon_{y y}^{o} \\ \gamma_{x y}^{o}\end{array}\right\}_{p}=\left\{\begin{array}{c}\varepsilon_{x x}^{o} \\ \varepsilon_{y y}^{o} \\ \gamma_{x y}^{o}\end{array}\right\}+z_{p}^{0}\left\{\begin{array}{c}k_{x} \\ k_{y} \\ k_{x y}\end{array}\right\}$

By Combining of (22) and (25) the strain components at any layer within a sub laminate are found in the form of

$\left\{\begin{array}{l}\varepsilon_{x x} \\ \varepsilon_{y y} \\ \gamma_{x y}\end{array}\right\}=\left\{\begin{array}{c}\varepsilon_{x x}^{o} \\ \varepsilon_{y y}^{o} \\ \gamma_{x y}^{o}\end{array}\right\}+\left(z-z_{p}^{0}\right)\left\{\begin{array}{c}k_{x} \\ k_{y} \\ k_{x y}\end{array}\right\}$

and the in-plane and shear stresses for any lamina of the $p^{\text {th }}$ sub laminatecan expressed as [19]

$\left\{\begin{array}{c}\sigma_{x} \\ \sigma_{y} \\ \sigma_{x y}\end{array}\right\}=\left[\begin{array}{lll}\bar{Q}_{11} & \bar{Q}_{12} & \bar{Q}_{16} \\ \bar{Q}_{21} & \bar{Q}_{22} & \bar{Q}_{26} \\ \bar{Q}_{16} & \bar{Q}_{26} & \bar{Q}_{66}\end{array}\right]\left\{\begin{array}{c}\varepsilon_{x} \\ \varepsilon_{y} \\ \varepsilon_{x y}\end{array}\right\}_{p}$
$\left\{\begin{array}{ll}\tau_{x z} \\ \tau_{y z}\end{array}\right\}=\left[\begin{array}{ll}\bar{Q}_{44} & \bar{Q}_{45} \\ \bar{Q}_{54} & \bar{Q}_{55}\end{array}\right]\left\{\begin{array}{l}\gamma_{x z} \\ \gamma_{y z}\end{array}\right\}_{p}$

By integrating equations 9 and 10 over the thickness of the sub laminate the elasticity matrix of the $p^{\text {th }}$ sub laminateis given by

$[\dot{D}]_{p}=\left[\begin{array}{ccc}\dot{A}_{i j} & z_{p}^{0} \dot{A}_{i j}+\dot{B}_{i j} & 0 \\ \dot{B}_{i j} & z_{p}^{0} \dot{B}_{i j}+\dot{D}_{i j} & 0 \\ 0 & 0 & \dot{A}_{s i j}\end{array}\right]_{p}$

Where $[\mathrm{A}],[\mathrm{B}]$, and $[\mathrm{D}]$ are the extension, bending extension coupling, and bending stiffness coefficients of the composite laminate, respectively.

$\left[A_{i j}\right]_{p}=\int_{-h / 2+z_{p}^{0}}^{+h / 2+z_{p}^{0}}[\bar{Q}] d z$ 


$$
\begin{aligned}
& {\left[B_{i j}\right]_{p}=\int_{-h / 2+z_{p}^{0}}^{+h / 2+z_{p}^{0}}[\bar{Q}]\left(z-z_{p}^{0}\right) d z } \\
&=\int_{-h / 2+z_{p}^{0}}^{+h / 2+z_{p}^{0}}[\bar{Q}] z d z-z_{p}^{0}\left[A_{i j}\right]_{p} \\
& {\left[D_{i j}\right]_{p}=\int_{-h / 2+z_{p}^{0}}^{+h / 2+z_{p}^{0}}[\bar{Q}]\left(z-z_{p}^{0}\right)^{2} d z \quad, \mathrm{i}, \mathrm{j}=1,2,6 } \\
& {\left[A_{s i j}\right]_{p}=\int_{-h / 2+z_{p}^{0}}^{+h / 2+z_{p}^{0}}[\bar{Q}] d z \quad \mathrm{i}, \mathrm{j}=4,5 }
\end{aligned}
$$

\section{PARTICLE SWARM OPTIMIZATION METHOD}

Optimization is the mechanism by which one finds the maximum or minimum value of a function or process. Inverse problems often occur in many branches of engineering where the values of some physical model parameters must be obtained from observed data. System identification for structural damage detection and health monitoring comes under the category of inverse problems[20]. The Particle swarm optimization (PSO) is a population based continuous optimization technique developed by Eberhart and Kennedy, can be used for solving such inverse problems. The system is initialized with a population of random solutions that constitute a swarm moving around in the search space looking for optima by updating generations. During each iteration of the algorithm, individual, or particle (the candidate solution) is evaluated by the objective function being optimized to determining the fitness of that solution.

At each iteration of the search procedure, the particle moves and updates its velocity and its position in the swarm based on experience and the results found by the particle itself, its neighbors and the swarm. It therefore combines three components ; its own current velocity, its best position $P_{i d}$ and the best position obtained by its informants or by the swarm $P_{g d}$. The research process is based on two rules:

- Each particle has a memory which can store the best position in which it has already passed and it tends to return to that position.

- Each particle is informed by the best known position within its neighborhood and it will tend to

move towards this position. The basic PSO algorithm consists of the velocity and position equation[21].

$$
\begin{aligned}
& \begin{array}{l}
V_{i d}^{k+1}=W_{i} \cdot V_{i d}^{k}+C_{1} \cdot \operatorname{rand}_{1}^{k} \cdot\left(P_{i d}-X_{i d}\right) \\
\quad+C_{2} \cdot \operatorname{rand}_{2}^{k} \cdot\left(P_{g d}-X_{i d}\right)
\end{array} \\
& \begin{array}{l}
X_{i d}^{k+1}=X_{i d}^{k}+V_{i d}^{k+1} \\
(30)
\end{array}
\end{aligned}
$$

where the superscript $\mathrm{i}$ denotes the particle and the subscript $\mathrm{k}$ denotes the iteration number, $W$ is the inertia weight, $\mathrm{C}_{1}$ and $\mathrm{C}_{2}$ are the acceleration constants, both taking values around 2 in general cases, constants $\operatorname{rand}_{1}^{\mathrm{k}}$ and $\mathrm{rand}_{2}^{\mathrm{k}}$ are random values in the range [0,1]; $\mathrm{P}_{\text {id }}$ is the neighboring best position occupied by the particle $\mathrm{i}$, and $\mathrm{P}_{\mathrm{gd}}$ is the global best position of the whole group.
Large values for $W$ favor exploration, which is the ability to explore regions of the search space, whereas small values promote (enhance, confirm, reinforce, sustain) exploitation, defined as the ability to concentrate the search around a promising area to refine a candidate solution. Besides, low values for $c 1$ and $c 2$ promotes a smooth particle trajectories, whereas high values favor more acceleration and abrupt movements. Therefore, small values for these optimization parameters can yield a slow convergence, while high values can yield a premature convergence[22].

The inertia term $w$ is adjusted dynamically during the optimization (decreased over the run), this will provide a balance between exploration and exploitation, [23].

$W_{i=} W_{\max }-k\left(W_{\max }-W_{\min }\right) / k_{\max }$

(31)

Where

$W_{\text {max }}$ is the initial weight factor,

$W_{\text {max }}$ is the final weight factor,

$\mathrm{k}$ is the current iteration number, and

$k_{\max }$ is the maximum allowable number of iterations.

A large initial value of $\mathrm{W}$ result in a greater diversity of population at the beginning of the optimization so as to promote global exploration of the search space. In the present work, the parameters used in the PSO were: $\mathrm{P}=50$ (number of particles); $\operatorname{wmax}=0.9 ;$ wmin $=0.4 ; \mathrm{c} 1=0.7$ and $\mathrm{c} 2=0.7$. Fig.4 shows Flow chart of PSO and FE code optimization process

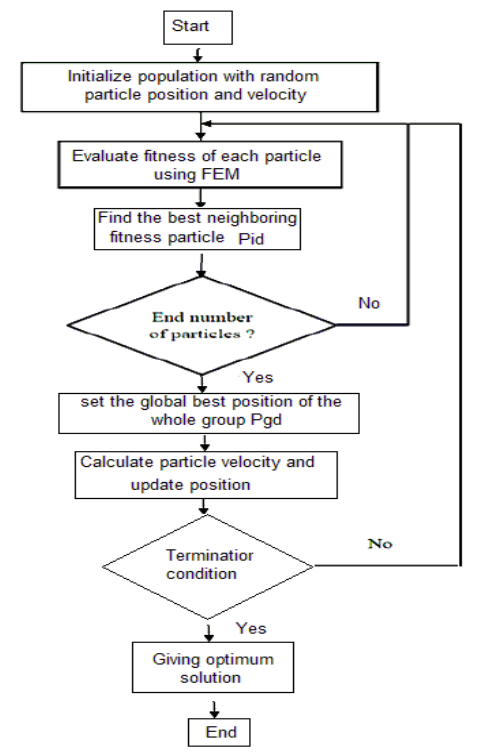

Fig 4: Flow chart of PSO and FE code optimization process.

\subsection{The Objective Function}

The damage assessment problem is reduced to a minimizing optimization problem by suitably assuming an objective function. 
For problems in which the variables are a combination of discrete and continuous variables, it is difficult to use conventional optimization algorithms such as the gradient based method, since they rely on the use of continuous variables. Natural frequencies are easier to measure than mode shapes and the error associated with its measurement is comparatively less. Hence, they are used. The objective function for the PSO optimization is formulated as follows:

$f_{\text {obj }}=\sum_{i=1}^{i=n} \operatorname{abs}\left(\omega_{\text {meas }}{ }^{2}-\omega_{\text {num }}{ }^{2}\right)_{i}$

where $n$ is the number of modes considered, $\omega_{\text {meas }}$ is the measured modal frequencies obtained either from experimental validation or from FE test cases (numerical validation) and $\omega_{\text {num }}$ is frequency determined by the fem model.

\section{RESULTS AND DISCUSSION}

\subsection{Evaluation}

The objective is to evaluate the feasibility of the proposed methods to identify and localize and quantities of damages in numerical models of the laminated composite plates with different boundary conditions.

A laminated composite plate made from glass fiber (E-Glass) is used as reinforcement in the form of woven fibers with epoxy resin as matrix for the composite plate. The numerical results for free vibration are presented for a laminated composite plate with delaminates have different locations and sizes.

The laminated composites considered for the present analysis are Simply supported boundary (SSSS), Clamped supported boundary conditions (CCCC), Clamped-Simply-ClampedSimply conditions (CSCS), and Clamped free clamped free conditions (CFCF). The boundary conditions are described as follows:

For Simply supported boundary, $\mathrm{S}$ where

$\mathrm{u}=\mathrm{v}=\mathrm{w}=\theta_{y}=0$ at $\mathrm{x}=0, \mathrm{a}$, and $\mathrm{u}=\mathrm{v}=\mathrm{w}=\theta_{x}=0$ at $\mathrm{y}=0, \mathrm{~b}$

and for Clamped boundary $\mathrm{C}: \mathrm{u}=\mathrm{v}=\mathrm{w}=\theta_{x}=\theta_{y}=0$ at $\mathrm{x}=0$, a or at $\mathrm{y}=0, \mathrm{~b}$

Based on the finite element formulation described earlier, the results are compared with the results of those published by other researchers and then study the influences of embedded delamination at different position and interface on the free vibration of composite structures.

To check the present delamination model, a composite plate made of E-glass fiber and epoxy resins has been considered for the comparison study. It has a total of eight layers of equal thickness which are arranged as $\left[0^{\circ} / 90^{\circ} / 0^{\circ} / 90^{\circ} / 90^{\circ} / 0^{\circ} / 90^{\circ} /\right.$ $\left.0^{\circ}\right]$.

The test plate has a size of $400 \mathrm{~mm}$ x $400 \mathrm{~mm}$ and total thickness $3.5 \mathrm{~mm}$. The composite plate has the delamination of $40 \mathrm{~mm} \times 40 \mathrm{~mm}$ size between $3^{\text {rd }}$ and $4^{\text {th }}$ layers from the top surface and its center located at the coordinate of $(275 \mathrm{~mm}$, $275 \mathrm{~mm}$ ) from the one corner of the plate.

The material properties of the plate are the density, $\rho=$ $1185 \mathrm{~kg} / \mathrm{m}^{3}$, the elasticity constants, $E_{1}=55 \mathrm{GPa}, E_{2}=$ $E_{3}=9.5 \mathrm{GPa}, G_{12}=G_{13}=5.5 \mathrm{GPa}, G_{23}=3.21 \mathrm{GPa}$, and the Poisson ratio $v_{12}=v_{13}=0.33, v_{12}=0.23$.
The results of the natural frequency for healthy and delaminated composite plates are shown in Table 1. From table, it can be found that an excellent agreement can obtained between the present method and those in [24]. These results indicate that the present FEM delamination model scheme is quite efficient.

Table 2. shows the results for frequency parameter of composite plate with different layer stacking sequences. From this table, it can be found that the present FEM can yield an acceptable level of agreement with those results obtained from ref[25], the first-order shear deformation theory (FSDPT) and the results from classical plate theory (CLPT) are listed.

Table 1. Validation of FEM for plate with free boundaries

\begin{tabular}{|c|c|c|c|c|}
\hline \multirow{2}{*}{$\begin{array}{c}\text { Mode } \\
\text { no: }\end{array}$} & \multicolumn{2}{|c|}{ Healthy Plate } & \multicolumn{2}{c|}{ Delaminated Plate } \\
\cline { 2 - 5 } & present & ABAQUS[24] & present & $\begin{array}{c}\text { ABAQUS } \\
{[24]}\end{array}$ \\
\hline 1 & 50.2628 & $50.03 \mathrm{~Hz}$ & 49.8571 & 49.96 \\
\hline 2 & 102.6525 & 101.42 & 101.2209 & 100.97 \\
\hline 3 & 135.8067 & 134.61 & 132.2840 & 134.21 \\
\hline 4 & 145.0167 & 143.55 & 143.0338 & 143.26 \\
\hline 5 & 168.8343 & 166.83 & 165.3161 & 166.80 \\
\hline 6 & 268.9648 & 265.87 & 265.0106 & 265.55 \\
\hline
\end{tabular}

Table 2. Comparison of frequencies from present study and $F E$ model of a simply supported laminated panels

$$
\bar{\omega}=\omega h \sqrt{\rho / E_{2}}
$$

\begin{tabular}{|c|c|c|c|c|}
\hline stacking & present & $\operatorname{Ref[25]}$ & $\begin{array}{c}\text { FSDPT }[2 \\
6]\end{array}$ & $\begin{array}{c}\text { CLPT[2 } \\
6]\end{array}$ \\
\hline$\left[\begin{array}{lllll}0 & 90 & 0 & 90\end{array}\right]$ & $\begin{array}{l}0.0698 \\
0.1639 \\
0.1639 \\
0.2264\end{array}$ & $\begin{array}{l}0.06826 \\
0.15747 \\
0.15747 \\
0.21400\end{array}$ & $\begin{array}{l}0.06791 \\
0.16066 \\
0.16066 \\
0.22097\end{array}$ & $\begin{array}{l}0.07474 \\
0.20737 \\
0.20737 \\
0.29824\end{array}$ \\
\hline [0 90] & $\begin{array}{l}0.0619 \\
0.1483 \\
0.1669 \\
0.2077\end{array}$ & $\begin{array}{l}0.06082 \\
0.14379 \\
0.14379 \\
0.19820\end{array}$ & $\begin{array}{l}0.06038 \\
0.14545 \\
0.14545 \\
0.20271\end{array}$ & $\begin{array}{l}0.06513 \\
0.17744 \\
0.17744 \\
0.25814\end{array}$ \\
\hline$\left[\begin{array}{lll}0 & 90 & 0\end{array}\right]$ & $\begin{array}{l}0.0713 \\
0.1329 \\
0.1670 \\
0.2265\end{array}$ & $\begin{array}{l}0.06882 \\
0.12823 \\
0.17532 \\
0.20835\end{array}$ & $\begin{array}{l}0.06931 \\
0.12886 \\
0.18674 \\
0.22055\end{array}$ & $\begin{array}{l}0.07769 \\
0.15185 \\
0.26599 \\
0.31077\end{array}$ \\
\hline
\end{tabular}

\subsection{Effect of delamination position and interface location on natural frequencies}

Presence of an embedded delamination leads to degradation in structural stiffness, which does not affect the mass distribution but reduces the stiffness of the structure and causes changes in modal parameters (frequencies, mode shapes, and modal damping factors). From the point of view of severity of a delamination, it is important to know, the size of the delamination, its shape and where it has occurred. So, in the present work, effect of these parameters on the natural frequencies has been studied in order to use for damage detection. It is clear that the natural frequencies are sensitive to damage and consequently to delamination five of the natural frequencies as features were chosen. 
Figure 5. explored the relations between the first five natural frequencies with different interface locations at a constant position (at center of plate) with $\alpha=\frac{A_{d}}{A}=0.04$ of total area of plate where $A_{d}$ and A are the plate and delamination areas.

Values of the material and geometric properties are as follows: The material properties of the plate are the density, $\rho=1185 \mathrm{~kg} / \mathrm{m}^{3}$, the elasticity constants, $E_{1}=55 \mathrm{GPa}$, $E_{2}=E_{3}=9.5 \mathrm{GPa}, G_{12}=G_{13}=5.5 \mathrm{GPa}, G_{23}=3.21 \mathrm{GPa}$, and the Poisson ratio $v_{12}=v_{13}=0.33, v_{12}=0.23$.

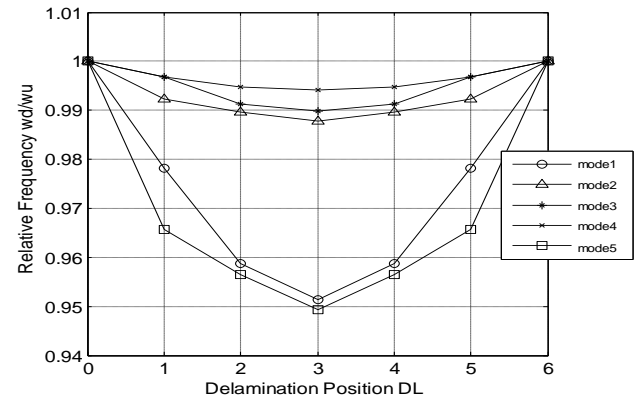

(a) SSSS

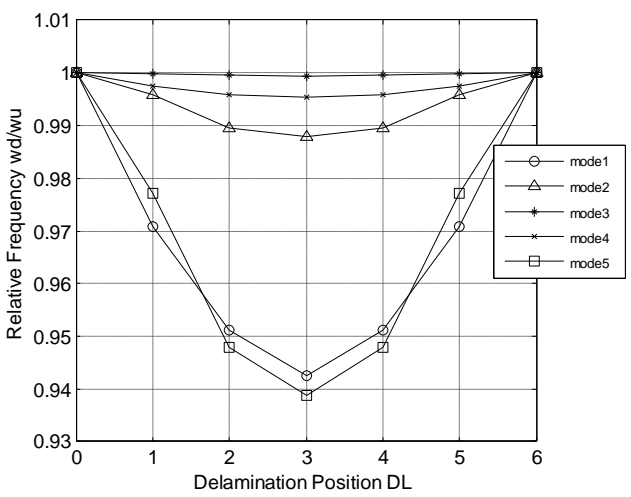

(c) CSCS
The results shown that the natural frequencies decreased with the existing of delaminate due to decreasing of the stiffness of the plate. And the amount of decrements varies with the fixing conditions, position of defect and the mode number. The maximum difference in frequency is in case CCCC at the position 3 and delaminate located at interface between layers 3 and 4. In SSSS, CCCC, and CSCS the $5^{\text {th }}$ mode is more effected with the presence of delamination while in case CFCF the $4^{\text {th }}$ is more effected.

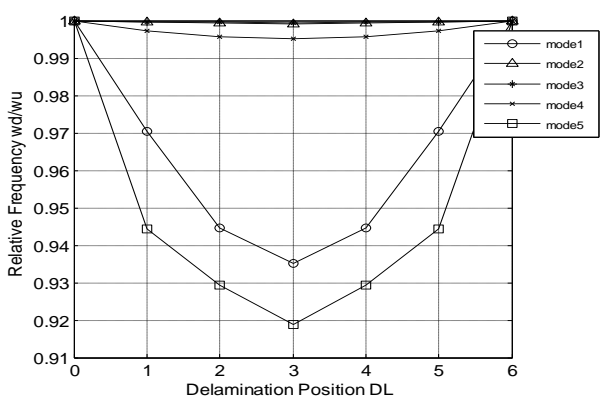

(b) $\mathrm{CCCC}$

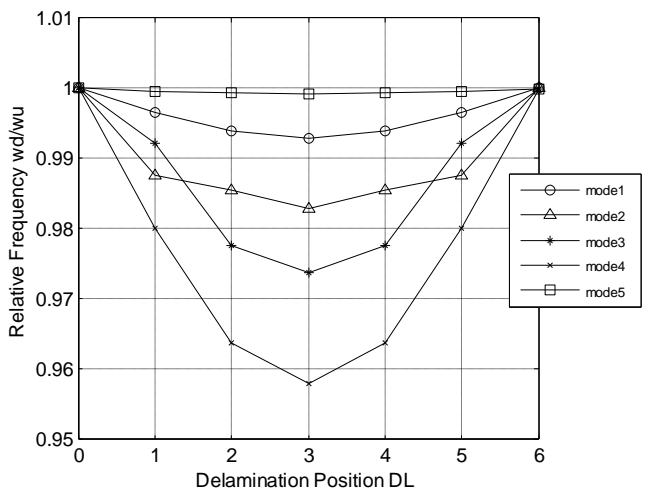

(d) CFCF

Fig 5: Effect of delamination interface location on relative natural frequencies for 1st-5th mode of SSSS, CCCC, CSCS, and CFCF BC's of 8 layer composite plate

The effect of delamination position on natural frequencies for 1 st to 5th mode of SSSS, CCCC, CSCS, and CFCF BC's of eight layer composite plate are shown in fig.6. The delaminate is embedded at interface between layer 3 and 4 . This delaminate is placed at position related to the element number as explained in figure7. In all cases studied, its found that delamination has more effect on the first

natural frequency especially for the case of CCCC. And also, it's found these effect varies with position and mode number.

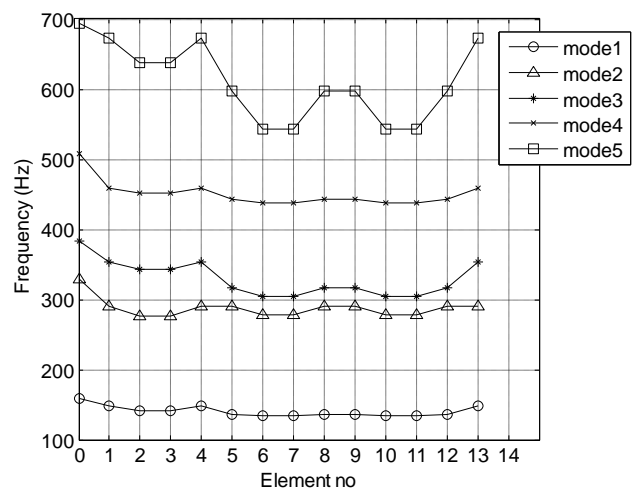

a)SSSS
From figures its can conclude that, the changes of the modal frequencies after delamination initiation, compared to those of a non-delaminated plate, gave a good indication of the existing of damage, demonstrating the feasibility of using measured changes in the vibration characteristics to detect damage.

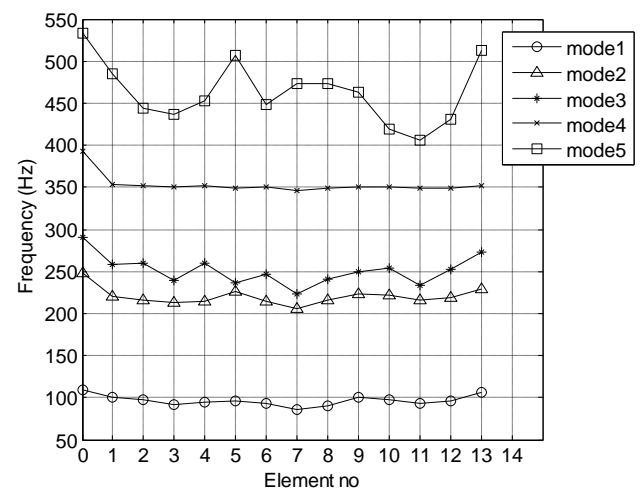

b) CCCC 


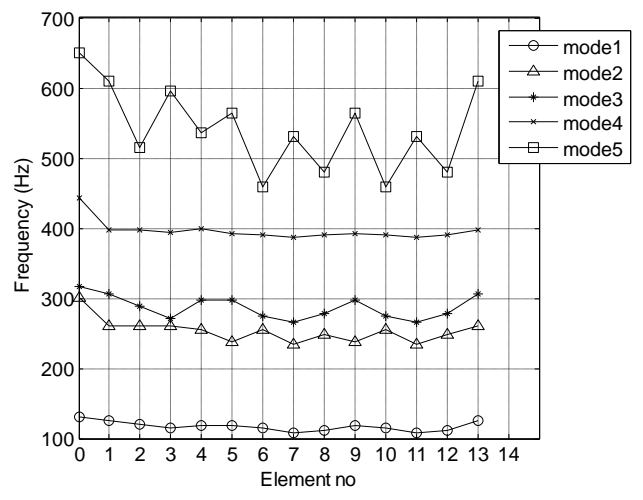

c)CSCS

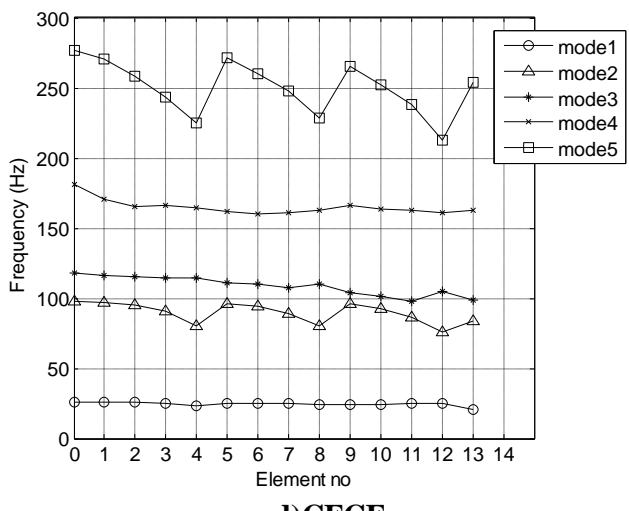

d) CFCF

Fig 6: Effect of delamination position on natural frequencies for $1 \mathrm{st}-5^{\text {th }}$ mode of SSSS, CCCC, CSCS, and CFCF BC's of $8^{\text {th }}$ layer composite plate.

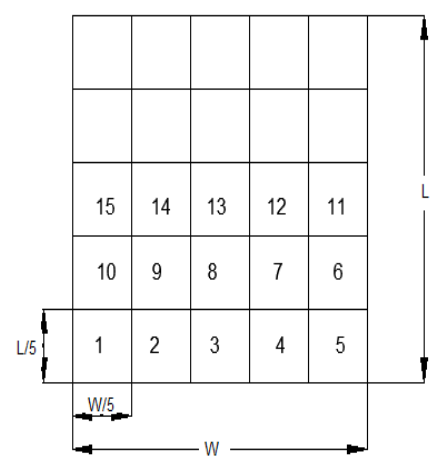

A further analysis, which results in terms of variation of the first four natural frequencies on the delamination area are also summarized in figure(8). In first frequency the variation is more depended on the supported ends while for higher modes, second, third, and fourth the variations are differing.

Fig 7: Delaminate position numbering.

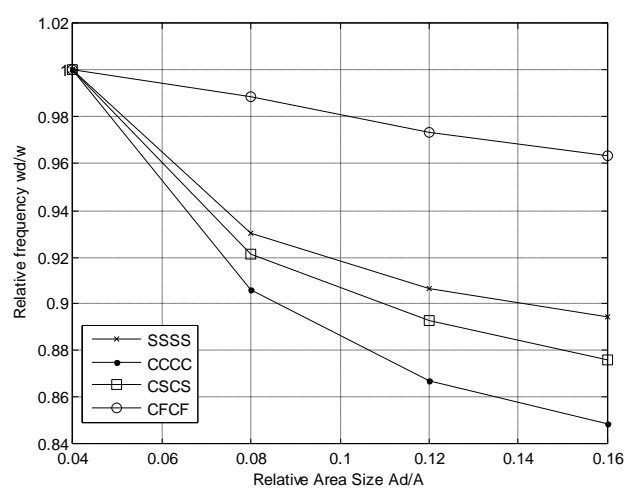

a) First mode

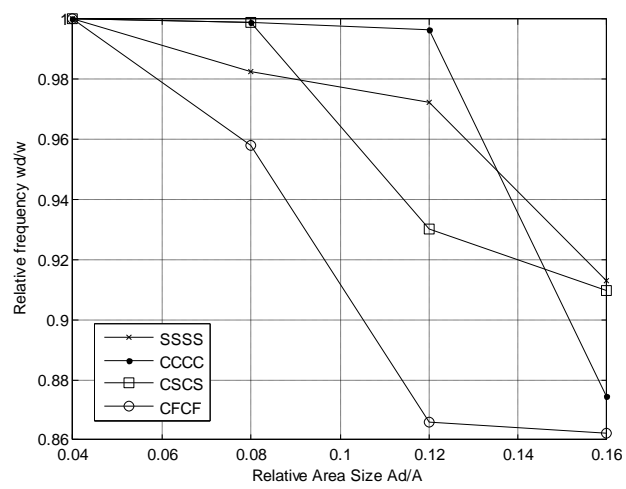

c) Third mode

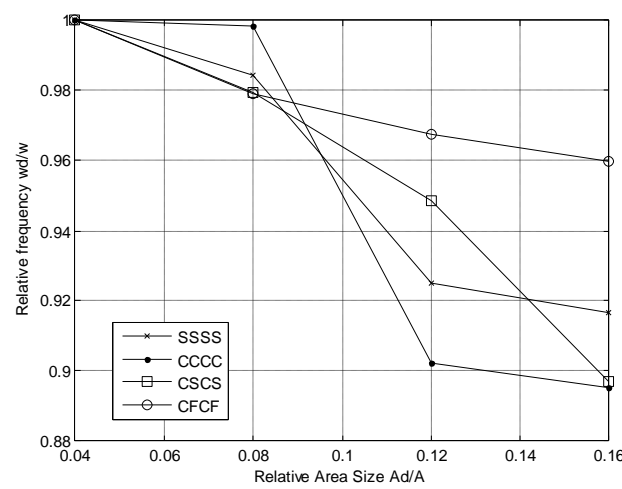

b) Second mode

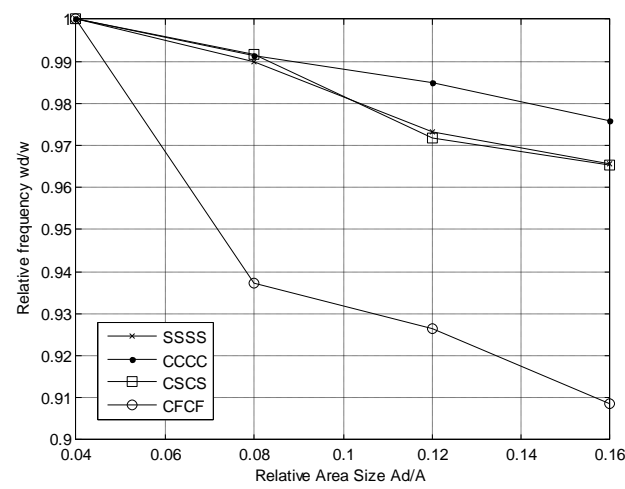

d) Fourth mode

Fig 8: Effect of delamination area on relative natural frequencies for the first four mode of SSSS, CCCC, CSCS, and CFCF BCs of of 8th layer composite plate. 


\subsection{Delamination Detection and Localization using PSO}

In this section, detection of the delamination, its position and its interface location using the natural frequencies is considered. The way to determine the occurrence of a delamination in the composite plate is discussed and explained in fig.(4).

A simple method for the delamination localization based on the PSO method is developed and applied to the delaminated plates of various boundary conditions.

As it is known, delamination results mainly in bending stiffness reduction then it can be expected to reduce the flexural natural frequencies of the structure [27] and in order to account this variation the first five natural frequencies is using in the evaluating (minimizing) of the objective function equation(32).

Table 3. Damage identification, position and interface location for composite plates using PSO

\begin{tabular}{|c|c|c|c|c|}
\hline & $X_{A C T}=[$ pos, layer,$\alpha]$ & $\operatorname{Predicted}\left(X_{P S O}\right)$ & Round $\left(X_{P S O}\right)$ & $f_{o b}$ \\
\hline \multirow[t]{5}{*}{ SSSS } & {$\left[\begin{array}{lll}1 & 3 & 0.08\end{array}\right]$} & {$\left[\begin{array}{llll}1.12 & 3.40 & 0.08\end{array}\right]$} & {$\left[\begin{array}{lll}1 & 3 & 0.08\end{array}\right]$} & \\
\hline & {$\left[\begin{array}{lll}1 & 1 & 0.08\end{array}\right]$} & {$\left[\begin{array}{lll}1 & 1 & 0.08\end{array}\right]$} & {$\left[\begin{array}{lll}1 & 1 & 0.08\end{array}\right]$} & $4.62 \mathrm{e}-05$ \\
\hline & {$\left[\begin{array}{lll}3 & 4 & 0.16\end{array}\right]$} & {$\left[\begin{array}{llll}3.00 & 3.88 & 0.16\end{array}\right]$} & {$\left[\begin{array}{lll}3 & 4 & 0.16\end{array}\right]$} & $4.58 \mathrm{e}-05$ \\
\hline & {$\left[\begin{array}{lll}8 & 5 & 0.16\end{array}\right]$} & {$\left[\begin{array}{llll}8.01 & 4.54 & 0.16\end{array}\right]$} & {$\left[\begin{array}{lll}8 & 5 & 0.16\end{array}\right]$} & $6.36 \mathrm{e}-05$ \\
\hline & {$\left[\begin{array}{lll}13 & 4 & 0.16\end{array}\right]$} & {$\left[\begin{array}{lll}12.56 & 3.73 & 0.16\end{array}\right]$} & {$\left[\begin{array}{lll}13 & 4 & 0.16\end{array}\right]$} & $1.30 \mathrm{e}-04$ \\
\hline \multirow[t]{5}{*}{$\mathrm{CCCC}$} & {$\left[\begin{array}{lll}1 & 3 & 0.08\end{array}\right]$} & {$\left[\begin{array}{lll}1.01 & 3.00 & 0.08\end{array}\right]$} & {$\left[\begin{array}{lll}1 & 3 & 0.08\end{array}\right]$} & $2.97 \mathrm{e}-05$ \\
\hline & {$\left[\begin{array}{lll}1 & 1 & 0.08\end{array}\right]$} & {$\left[\begin{array}{lll}1.06 & 1.00 & 0.08\end{array}\right]$} & {$\left[\begin{array}{lll}1 & 1 & 0.08\end{array}\right]$} & $2.38 \mathrm{e}-05$ \\
\hline & {$\left[\begin{array}{lll}3 & 4 & 0.16\end{array}\right]$} & {$\left[\begin{array}{lll}3.19 & 4.07 & 0.16\end{array}\right]$} & {$\left[\begin{array}{lll}3 & 4 & 0.16\end{array}\right]$} & $1.71 \mathrm{e}-05$ \\
\hline & {$\left[\begin{array}{lll}8 & 5 & 0.16\end{array}\right]$} & $\left.\begin{array}{llll}7.52 & 5.32 & 0.16\end{array}\right]$ & {$\left[\begin{array}{lll}8 & 5 & 0.16\end{array}\right]$} & $1.79 \mathrm{e}-05$ \\
\hline & {$\left[\begin{array}{lll}13 & 4 & 0.16\end{array}\right]$} & [13.09 4.300 .16$]$ & {$\left[\begin{array}{lll}13 & 4 & 0.16\end{array}\right]$} & $3.67 \mathrm{e}-05$ \\
\hline \multirow[t]{5}{*}{ CSCS } & {$\left[\begin{array}{lll}1 & 3 & 0.08\end{array}\right]$} & {$\left[\begin{array}{lll}1.08 & 2.97 & 0.08\end{array}\right]$} & {$\left[\begin{array}{lll}1 & 3 & 0.08\end{array}\right]$} & $7.41 \mathrm{e}-05$ \\
\hline & {$\left[\begin{array}{lll}1 & 1 & 0.08\end{array}\right]$} & {$\left[\begin{array}{lll}1.24 & 1.22 & 0.08\end{array}\right]$} & {$\left[\begin{array}{lll}1 & 1 & 0.08\end{array}\right]$} & $2.46 \mathrm{e}-05$ \\
\hline & {$\left[\begin{array}{lll}3 & 4 & 0.16\end{array}\right]$} & {$\left[\begin{array}{lll}3.43 & 4.22 & 0.16\end{array}\right]$} & {$\left[\begin{array}{lll}3 & 4 & 0.16\end{array}\right]$} & $3.81 \mathrm{e}-05$ \\
\hline & {$\left[\begin{array}{lll}8 & 5 & 0.16\end{array}\right]$} & {$\left[\begin{array}{lll}8.23 & 3.48 & 0.16\end{array}\right]$} & {$\left[\begin{array}{lll}8 & 5 & 0.16\end{array}\right]$} & $1.93 \mathrm{e}-04$ \\
\hline & {$\left[\begin{array}{lll}13 & 4 & 0.16\end{array}\right]$} & {$\left[\begin{array}{lll}13.08 & 3.57 & 0.16\end{array}\right]$} & {$\left[\begin{array}{lll}13 & 4 & 0.16\end{array}\right]$} & $6.51 \mathrm{e}-05$ \\
\hline \multirow[t]{5}{*}{$\mathrm{CFCF}$} & {$\left[\begin{array}{lll}1 & 3 & 0.08\end{array}\right]$} & $\left.\begin{array}{llll}1.00 & 3.27 & 0.08\end{array}\right]$ & $\left.\begin{array}{lll}1 & 3 & 0.08\end{array}\right]$ & $3.28 \mathrm{e}-05$ \\
\hline & {$\left[\begin{array}{lll}1 & 1 & 0.08\end{array}\right]$} & {$\left[\begin{array}{lll}1 & 1 & 0.08\end{array}\right]$} & {$\left[\begin{array}{lll}1 & 1 & 0.08\end{array}\right]$} & $2.96 \mathrm{e}-05$ \\
\hline & {$\left[\begin{array}{lll}3 & 4 & 0.16\end{array}\right]$} & {$\left[\begin{array}{lll}2.94 & 4.44 & 0.16\end{array}\right]$} & {$\left[\begin{array}{lll}3 & 4 & 0.16\end{array}\right]$} & $2.16 \mathrm{e}-05$ \\
\hline & {$\left[\begin{array}{lll}8 & 5 & 0.16\end{array}\right]$} & $\left.\begin{array}{lll}7.72 & 5.31 & 0.16\end{array}\right]$ & {$\left[\begin{array}{lll}8 & 5 & 0.16\end{array}\right]$} & $3.27 \mathrm{e}-05$ \\
\hline & {$\left[\begin{array}{lll}13 & 4 & 0.16\end{array}\right]$} & $\left.\begin{array}{llll}13.44 & 3.87 & 0.16\end{array}\right]$ & {$\left[\begin{array}{lll}13 & 4 & 0.16\end{array}\right]$} & $7.36 \mathrm{e}-05$ \\
\hline
\end{tabular}

\section{CONCLUSIONS}

This investigation considered the problem for delamination detection, localization and quantification in a composite plate. Parametric study on the effect of boundary conditions and interlaminate location and position of damage on a composite laminated plate was also investigated. It is found that the stiffness decreases with the increase of delamination area providing a reduction in natural frequencies. The analyses of show that the delamination induced changes of the plate parameters are mode-dependent and the effect of damage appears in earlier mode shapes.

Particle Swarm Optimization algorithm was used for identification of the presence of delamination and it is possible to locate and quantify its area.

Results show the procedure incorporating the FEM modeling of damage and PSO methods is an effective in the damage assessment of plate structures. Particle Swarm algorithm was found to be superior in convergence and accuracy.

The investigation shown that the localization was possible with a high confidence in all cases studied where no localization error. results for different boundary conditions and damage location
An eight-ply [0/90/90/0]s E-Glass/Polyester of size $240 \mathrm{~mm}$ x $240 \mathrm{~mm} \times 2.2 \mathrm{~mm}$ with the following material properties is used: $\rho=1200 \mathrm{~kg} / \mathrm{m}^{3}$, the elasticity constants, $E_{1}=E_{2}=$ $17.250 \mathrm{GPa}, \quad E_{3}=9.5 \mathrm{GPa}, G_{12}=2.91 \mathrm{GPa}, G_{13}=G_{23}=$ $2.795 \mathrm{GPa}$, and the Poisson ratio $v_{12}=v_{13}=0.43, v_{12}=$ 0.14 .

From table 3. it can be seen that for all boundary conditions the PSO success in predict the damage parameter $X_{A C T}=[\mathrm{pos}$, layer, $\alpha]$ after using the round command to get the nearest integers for $X$, where pos represent the delaminate position, layer define the interface layer for delamination location and $\alpha$ area ratio.
PSO with a two dimensional FEM delamination model can be used as a reliable model/tool in estimating of the severity of damage.

This again permits the development of an automatic process, using the response vibration signal, to locate and quantify the fault.

It is a very good achievement, because it is possible, with a few vibration modes can answer all the assessment questions mentioned above.

From the outcome of the present work, it is felt that the use of PSO in detecting embedded delamination size, position and interlaminar damage location in an FRP composite laminated structure is a promising area.

Finally, it is concluded that PSO method and FEM delamination model to composite plate provides sensitive, reliable and accuracy on damage assessments.

\section{REFERENCES}

[1] Obinna, K.I . 2012. Vibration based damage detection in composite structures using computational intelligence tools, Thesis ,University of New South Wales Australian Defence Force Academy. 
[2] Zheng, J.Q. Zheng, L. and Hong, T.W. 2011. A genetic fuzzy radial basis function neural network for structural health monitoring of composite laminated beams. Expert Systems with Applications, 38:11837-11842.

[3] VENNA, V. W. 2012. Delamination effect on response of a composite beam by wave plate spectral finite element method. Thesis, National institute of technology, Rourkela, Orissa.

[4] Rytter, A. and Kirkegaard, P.H. 1993. The Use of neural networks for damage detection and location in steel member," Third international conference on the application of artificial intelligence of civil engineering, Edinburgh.

[5] Gomes, H.M., and Silva, N.R.S. 2008. Some comparisons for damage detection on structures using genetic algorithms and modal sensitivity method. Applied Mathematical Modeling,. Vol. 32, p. 22162232

[6] Ali,Y., and Krishna, S. Structural Health Monitoring of Composites using System Identification," 15th Australian International Aerospace Congress (AIAC15).

[7] Shen,S. Gun,J.Y. and Pizhong, Q. 2010. Delamination identification of laminated composite plates using a continuum damage mechanics model and subset selection technique, Smart materials and structures. 19, 055024 (13pp).

[8] Vinh,P. N., Pierre,K., and Stephane, P.A. 2013. Isogeometric cohesive elements for two and three dimensional composite delamination analysis. Elsevier Science, Journal of Composites: Part B, Engineering.

[9] Seth,S.K. and etal. 2002. Damage detection in composite materials using frequency response methods. science direct, Composites Part B: Engineering Volume 33, Issue 1, January, Pages 87-95.

[10] Kyoung, T.K. and et al. 2011. Damage detection of composite plates using finite element analysis based on structural health monitoring," Journal of Materials Science and Engineering B1,14-21.

[11] Oruganti,K. Mehdizadeh,M. John, S. and Herzberg, I. 2008. Damage detection in composites using vibration signatures," Conference: Proceedings of the International Conference on Scientific Computing, CSC, Las Vegas, Nevada, USA.

[12] Nasiri,M.R. Mahjoob, M.J. and Aghakasiri, A. 2011. Damage Detection in a Composite Plate Using Modal Analysis and Artificial Intelligence. Appl. Compos Mater $18: 513-520$

[13] Ferdinando, A. and Elio,S. 1999. A mixed enhanced finite element for the analysis of laminated composite plates. Int. J. Numer. Meth. Engng. 44, 1481-1504.

[14] Venkata, S. K. 2007. Static analysis of cross-ply laminated composite plate using finite element method. Thesis, Department of mechanical engineering national institute of technology, Rourkela.
[15] Parhi,P.K. Bhattacharyya, S.K. and Sinha, P.K. 2001 Failure analysis of multiple delaminated composite plates due to bending and impact. Bull. Mater. Sci., Vol. 24, No. 2, pp. 143-149. (C) Indian Academy of Sciences.

[16] Sudip,D. Ankuran, S. and Amit, K. 2011. Free vibration analysis of bending stiff composite conical shells with delamination-A finite element approach. Proceedings of the International Conference on Mechanical Engineering (ICME2011) 18-20, Dhaka, Bangladesh.

[17] Gim, C.K. Plate finite element modeling of laminated plates. Composite Structure, Vol. 52, pp.157-168.

[18] Wang,S. and Dawe,D.J. 2002. Dynamic instability of composite laminated rectangular plates and prismatic plate structures. Elsevier Science, Comput. Methods Appl. Mech. Engrg. 191,1791-1826

[19] Sandesh, S. and Krishnapillai, S. 2011. Structural damage detection using a hybrid particle swarm algorithm. World Journal of Modeling and Simulation Vol. 7, No. 4, pp. 290-298.

[20] SAID, L. AMIRA,G. and SALIM,C. A modified hybrid particle swarm optimization algorithm for solving the traveling salesmen problem. 2012. Journal of Theoretical and Applied Information Technology, Vol. 39 No.2.

[21] Rosilene, A.P. Leonardo, T.S. and Roberto,A.T. 2013. Damage identification in plates via flexibility matrix from noisy data using the particle swarm optimization method," DINAME - Proceedings of the XV International Symposium on Dynamic Problems of Mechanics M.A. Savi (Editor), ABCM, Buzios, RJ, Brazil, February 17-22.

[22] Chen, J. Peng, Ge, W. R. and Wei, J. 2009. Optimal design of composite laminates for minimizing delamination stresses by particle swarm optimization combined with FEM. Structural Engineering and Mechanics, Vol. 31, No. 4, 407-421.

[23] Israr, U. and Jyoti, K.S. 2013. A Novel Method for Delamination Detection in Composites," International Journal of Innovation and Applied Studies ISSN 20289324 Vol. 2 No. 1 Jan., pp. 34-42.

[24] N Hu, H Fukunaga, M Kameyama, and Y Aramaki, 2002. Vibration analysis of delaminated composite beams and plates using a higher-order finite element," International Journal of Mechanical Sciences 44,14791503.

[25] Nosier, A. Kapania, R.K. and Reddy,J.N. 1993. Free vibration analysis of laminated plates using a layerwise theory. AIAA Journal;31(12):2335-46.

[26] Park, T. Lee, S.Y. and Voyiadjis, G.Z. 2009. Finite element vibration analysis of composite skew laminates containing delaminations around quadrilateral cutouts. Elsevier Science, Composites: Part B, 40,225-236. 